\title{
Analysis of the Diversity of Yeast Communities in Xinjiang Honey
}

\section{Yumei Wei}

Shihezi University

\section{Yonghui Lei}

Shihezi University

Ailing Liu

Shihezi University

Shanshan Zhu

Shihezi University

Yang Li

Shihezi University

Yanli Cai

Shihezi University

Yanfei Sun (D81711308@qq.com )

Shihezi University https://orcid.org/0000-0003-1947-9245

\section{Research Article}

Keywords: raw honey, yeast, 26s rRNA sequencing, diversity

Posted Date: March 7th, 2022

DOI: https://doi.org/10.21203/rs.3.rs-1349085/v1

License: (c) (1) This work is licensed under a Creative Commons Attribution 4.0 International License.

Read Full License 


\section{Abstract}

Honey is widely produced in China as a natural health food with high nutritional value. In this study, highthroughput sequencing (HTS) and culture-dependent methods were used to assess the yeast diversity of wild honey from three different regions of Xinjiang. The analysis of HTS data of 26S rDNA NL1F and NL2R region genes revealed the highest number of operational taxonomic units (OTUs) of the yeast genus Zygosaccharomyces in honey, followed by the genera Aureobasidium and Clavispora. Multifloral honey is not only different in yeast structure and composition from monofloral honey, but also more diverse than monofloral honey. Thirty-five yeast strains were obtained by traditional isolation and culture methods, and morphological, physiological and biochemical, and 26S rDNA D1/D2 domain genes were sequenced to identify 11 yeast species, including Zygosaccharomyces siamensis, Aureobasidium pullulans, Naganishia albida, Saccharomyces cerevisiae, Wickerhamomyces anomalus, Rhodotorula glutinis, Pichia kluyveri, Barnettozyma californica, Rhodotorula kratochvilovae, Candia tropicalis, Pichia terricola, and these results are not entirely consistent with those obtained using high-throughput sequencing methods. Different species of honey may have contributed to the diversity of yeast. The results of this study provide basic information on the yeast diversity of native honey in Xinjiang, which will help to develop germ plasm resources of yeast in honey.

\section{Introduction}

Honey is a natural sweet substance collected by bees from plant nectar, secretions or the honeydew, mixed with their own secretions(Roshan et al. 2017; Danila et al. 2018). Honey is rich in nutrients, including sugars, proteins, amino acids, vitamins, organic acids, trace elements and some biological enzymes(Meo et al. 2017; Shapla et al. 2018). Depending on the nectar plant, honey can be divided into monofloral honey, which is honey made from the nectar or secretions of one or more nectar plants, and multifloral honey, which is honey made from the nectar or secretions of two or more nectar plants. In addition, the composition of monofloral honey and multifloral honey may differ, such as $680.2 \mathrm{mg} / \mathrm{g}$ of fructose and glucose in Oleaster honey(Zhu 2021), $812 \mathrm{mg} / \mathrm{g}$ of fructose and glucose in sunflower honey (Zhao et al. 2015), and $765.6 \mathrm{mg} / \mathrm{g}$ of fructose and glucose in multifloral honey(Zhao et al. 2015). 4Terpineol is the most significant characteristic volatile substance of sunflower honey(Su et al. 2010), Oleaster honey contains the most phenolic substances, while multifloral honey contains the most aldehydes(Di et al. 2016; Wei et al. 2020).

Generally speaking, most honey contains high sugar content and low water content, which is extremely unsuitable for microorganisms. Thus, the maximum level allowed by this legislation (commercial honey ruled by the Mercado Común del Sur (MERCOSUR) legislation) for molds and yeasts with trading purposes is $100 \mathrm{CFU} / \mathrm{g}$ of honey (A et al. 2017). But so far, whether the yeast in honey is detrimental to health is still unknown(Devi et al. 2021). Some people have reported that yeast that produces erythritol from honey has been found (Deng et al. 2013) ethanol-producing yeast (Joseph et al. 2018), lipaseproducing yeast (Palilu et al. 2019), but yeast can also affect the quality of honey (Hajimohammadi et al. 2017). Therefore, we are quite interested in the biodiversity of yeast in honey. 
Yeast is an important industrial microorganism and has important applications in the fields of food, feed, medicine and environmental management(Huang et al. 2020). Screening excellent yeast strains in honey is of great significance for products with regional characteristics and unique flavors in Xinjiang. Rering Caitlin $C$ et al. found that pollen and yeast may work together to alter the chemical composition of nectar, thereby affecting honey quality(Rering et al. 2021). Echeverrigaray Sergio et al. isolated yeast from 17 stingless bee honey samples in southern Brazil and found that some yeasts were only identified in honey samples from specific bee species, indicating that these yeasts are closely related to insects(Echeverrigaray et al. 2021), and the isolated yeast species exhibited high osmotic pressure (Palilu et al. 2019)and low sugar assimilation (Park et al. 1996)capacity. Osmophilic yeasts such as Yong Kun Park isolated from honey and pollen not only convert sucrose to polymers but also to fructooligosaccharides in good yields. It has been reported that osmophilic yeast produce lipase due to their low water content in their natural habitat. Palilu Prayolga Toban (Palilu et al. 2019)Lipase-producing yeast isolated from wild forest honey in Sulawesi. Xinjiang is a traditional beekeeping province with an extensive mass beekeeping base, and it has a typical continental climate with large diurnal temperature differences(ZHANG et al. 2020) providing unique conditions for the formation and evolution of species(Zhu et al. 2021). By studying the community structure of yeast in honey, the germplasm resources of yeast in honey were developed and utilized.

The ultimate goal of this study was to determine the basic structure of the honey yeast community suitable for the special environmental characteristics of Xinjiang. High-throughput sequencing techniques and culture-dependent methods were combined for accurate identification. The reported data will be useful for the study of yeast diversity in honey and as a guide for the development and utilization of yeast germ plasm resources.

\section{Materials And Methods}

\section{Sample Collection}

Honey was collected from Shawan, Shihezi and Yili Kazakh Autonomous Prefecture in Xinjiang, and all samples were obtained directly from beekeepers during the flowering season. The samples are kept fresh in the sampling box and shipped to the laboratory. Honey samples for cultural isolation were stored at $4^{\circ} \mathrm{C}$, and genome-extracted honey samples for high-throughput sequencing were stored at $-80^{\circ} \mathrm{C}$. There were 3 samples of Oleaster honey ( 3 from Shawan area), 3 samples of sunflower honey ( 3 from Shihezi area), and 3 samples of multifloral honey ( 3 from lli area).

\section{High-throughput sequencing}

Three replicates of each sample were taken and mixed together in equal amounts(Zhang et al. 2021). Using the MoBio Power Fecal ${ }^{8}$ DNA Isolation Kit (MOBIO, USA), total DNA was extracted directly from each sample according to the manufacturer's protocol. The yeast 26S rDNA was amplified with a pair of specific primers with barcode NL1F (forward primer) (5'-GCAT ATCAATAAGCGGAGGAAAAG-3') and NL2R (reverse primer) (5'-CTTGTTCGCTATCGGTCTC-3') (Liu et al. 2018). The PCR reaction system (20 $\mu \mathrm{L})$ 
contained 5x FastPfu Buffer $(4 \mu \mathrm{L}), 2.5$ mM dNTPs $(2 \mu \mathrm{L})$, primer (5 $\mu \mathrm{M} ; 0.8 \mu \mathrm{L}$ each), FastPfu Polymerase $(0.4 \mu \mathrm{L}), \mathrm{BSA}(0.2 \mu \mathrm{L})$, and template DNA (10 ng). (Zhu et al. 2021). The PCR reaction was performed using a thermocouple PCR system as follows: 5 min at $95^{\circ} \mathrm{C}$ (denaturation), 30 cycles at $95^{\circ} \mathrm{C}$ for $1 \mathrm{~min}, 55^{\circ} \mathrm{C}$ for $30 \mathrm{~s}$, and $72{ }^{\circ} \mathrm{C}$ for $1 \mathrm{~min}$, and finally, at $72{ }^{\circ} \mathrm{C}$ for $10 \mathrm{~min}$ (elongation). The PCR products were analyzed using $2 \%$ agarose gel electrophoresis, purified using the AxyPrep DNA Gel Extraction Kit (Axygen Biosciences, USA).The DNA fragments were quantified using QuantiFluor ${ }^{\mathrm{TM}}$-ST (Promega, USA) (Zhang et al. 2018). Equimolar amounts of purified DNA fragments were pooled after individual samples were tagged with indexes through an index PCR, and the Illumina MiSeq PE2500 platform (Illumina, USA) was used to perform paired-end sequencing $(2 \times 250)$ following the protocol by Personalbio Biomedical Technology (Shanghai, China).

Raw reads are prime clipped and paired reads are combined into a single sequence. The data for each sample are identified and distinguished by barcodes. They then quality filter using QIIME (version 1.8.0). All sequences were clustered based on the distance between sequences. Sequences were assigned to operational classification units (OTUs) based on 97\% similarity(Zhao et al. 2011). The RDP Classifier method was adopted to annotate the species of OTU representative sequence (threshold is $0.8-1$ ), and the yeast diversity of samples was counted at the classification level of domain, phylum, class, order, family, and genus(Zhang et al. 2021). Excel 2007 (Microsoft Corp., United States) was used for yeast species abundance (mean values)(Li et al. 2019). Origin Pro 2018 (OriginLab., United States) was used to make a heatmap of yeast species in honey. Raw fungal ITS sequence data were deposited at the NCBI database under accession number SUB10550652.

\section{Yeast isolation and cultivation}

Yeasts were isolated from honey samples by published method under aseptic conditions(Boundy-Mills 2006). Honey was diluted by 10 being with sterile water and vortexed for 1 minute(P et al. 2003). One hundred microliters of successive decimaldilutions were spread on yeast extract-Malt extract (YM)agar (Qingdao Hope Bio-Technologyn Co.,Ltd)supplemented with 100mg l-1 chloramphenicol(Saksinchai et al. 2012). A representative purified yeast colony was selected from each sample, and each colony was streaked on Wallerstein Laboratory (WL nutrient agar) Nutrient Agar(ShanDong Tuopu Biol-engineering Co.,Ltd) to better identify their morphological type (Pallmann et al. 2001). Then store them in YM slope or $30 \%$ (v/v) glycerin.

\section{Physiological and biochemical identification}

The composition of all media used in this study and the methods of fermentation and assimilation tests were referred to Kurtzman(Kurtzman and Cletus 2011; Kurtzman et al. 2011; Xu et al. 2019). Both sugar fermentation and assimilation tests of the yeast were performed at $25^{\circ} \mathrm{C}$ and recorded after $1,2,3$ weeks(S et al. 2013).

(1) Sugar fermentation test: The sugar to be fermented (e.g. glucose, sucrose, maltose, lactose) was added to a $0.6 \%$ solution of yeast extract to bring the sugar content to $2 \%$, and then the prepared solution 
was added separately to test tubes containing Duchenne tubes, and the activated test strain was accessed and incubated in a constant temperature incubator at $28^{\circ} \mathrm{C}$ for 3 weeks. During incubation, if bubbles appear at the top of the Duchenne tube, the test strain can use the sugar, which can be recorded as "+" (positive reaction), if there is no change, it is recorded as "-" (negative reaction).

(2) Carbon source assimilation test: The test strain in the growth phase was inserted into the test tube containing carbon source liquid mediumc(Xu et al. 2019) and incubated in a constant temperature incubator at $28^{\circ} \mathrm{C}$ for 3 weeks to observe the growth and the change of the solution, observe the growth and the change of the solution, and control with the blank test to observe whether the solution is turbid, whether the formation of atolls and bacteria Pu.

(3) Nitrogen source assimilation test: the test strains in the growth period into the nitrogen source liquid medium, incubated at $28^{\circ} \mathrm{C}$ for 3 weeks in a constant temperature incubator, observe the growth and solution changes as in the carbon source assimilation test.

\section{Molecular biology identification}

In the experiment, acetyl trimethyl ammonium bromide (CTAB) method was utilized to extract yeast genomic DNA ( $M$ et al. 2010). PCR amplification of yeast 26S rRNA D1/D2 sequences using fungal universal primers s NL1 (GCATATCAATAAGCGGAGGAAAAG) and NL4 (50 GGTCCGTGTTTCAAGACGG30) (úbeda et al. 2014; $\mathrm{H}$ Tansel Yalçın 1 2021). PCR reaction was performed as described by Wang and Liu (2013) with a fiducial volume of $50 \mu \mathrm{L}$ mixture containing $0.2 \mathrm{mmol} / \mathrm{L}$ of each $\mathrm{dNTP}, 1 \times$ buffer without $\mathrm{Mg} 2+, 1.5 \mathrm{mmol} / \mathrm{L} \mathrm{MgCl} 2,0.2 \mu \mathrm{mol} / \mathrm{L}$ of each primer, $1.5 \mathrm{U}$ Taq polymerase (TaKaRa, Dalian, China), and $1 \mu \mathrm{L}$ DNA template (Wang et al. 2019). The amplification procedure: pre-denaturation at $94{ }^{\circ} \mathrm{C}$ for $5 \mathrm{~min}$; denaturation at $94{ }^{\circ} \mathrm{C}$ for $45 \mathrm{~s}$, annealing at $55^{\circ} \mathrm{C}$ for $45 \mathrm{~s}$, extension at $72{ }^{\circ} \mathrm{C}$ for 1 min, 30 cycles; extension at $72{ }^{\circ} \mathrm{C}$ for another $10 \mathrm{~min}$, store at $4{ }^{\circ} \mathrm{C}$. The quality of the PCR products was verified by GeneGreen-stained $1 \%$ agarose gel electrophoresis at $120 \mathrm{~V}$ for $30 \mathrm{~min}$. The PCR products were directed to Shanghai Biotech for purification and sequencing. The sequences analyzed in this study were blasted in the NCBI database (http://www.ncbi Im.nigh.gov/BLAST), and the registration numbers of the yeast strains are shown in Table 6 and compared with those in the NCBI's GenBank database.

\section{Results}

\section{Determination of yeast community structure distribution by high-throughput methods}

In order to characterize the structure of the yeast community in honey from three regions of Xinjiang, the OTU sequences were clustered and annotated with species. 40 genera belonging to 2 phyla, 10 orders, 13 families and 15 families were included in the OTU of honey from the three sampling sites. The top 20 genera in relative abundance and the lower genera in relative abundance were selected and grouped into others. Zygosaccharomyces, Aureobasidium, Clavispor, Pichia and Filobasidium were the main yeast genera in honey. The results for the main yeast genera levels in honey (Fig.1) showed that Zygosaccharomyces had the highest relative abundance of $85.86 \%$ and was the most dominant 
genus among the three honey, followed by Aureobasidium and Clavispora. And the top 20 genera were distributed in all three honey.The relative abundances of Oleaster honey and sunflower honey differed little at the yeast genus level, but there were large differences in the dominant yeast and their relative abundances with multifloral honey. The highest relative abundance of Zygosaccharomyces was found in sunflower honey at the Shihezi sampling site (96.64\%), followed by Clavispora (1.24\%) and Filobasidium $(1.06 \%)$, while the relative abundance of Zygosaccharomyces reached $(44.71 \%)$ in multifloral honey at the lli sampling site, and followed by Aureobasidium (14.07\%) and Pichia (9.80\%).

Heat map analysis showed the relative abundance of the last 20 yeast genera in the three honey groups (Figure 2). The relative abundance of these yeast genera was generally low in all three samples. Among them, the genus Barnettozyma was present in FM1 and FM3 yeast, but no tested were available in FM2. Eremothecium was present in both FM2 and FM3, but not in FM1.

\section{Morphological identification of yeast}

Through isolation and purification of YM medium, 15, 13 and 7 yeast strains were isolated and purified from Shawan oleaster honey, Shihezi sunflower honey and Yili multifloral honey, and a total of 35 yeast strains were obtained (Table 1). According to the phenotypic characteristics of yeast on WL medium, it can be classified into 11 different phenotypes (Figure 3), and then, the cell morphology of each phenotypic representative strain was observed, a total of three microscopic forms were obtained. Singleended sprouting of pike (phenotype FM1-1), round single end sprouting (phenotype FM1-2), Oval singleended sprouts (phenotype FM1-3,FM1-4,FM1-8,FM1-10,FM1-12,FM2-10,FM3-1,FM3-3,FM3-7).

\section{Physiological and biochemical experiments}

Based on morphological characterization, further physiological and biochemical experiments were conducted on yeast, including sugar fermentation experiments, carbon source assimilation experiments, nitrogen source assimilation experiments, and maximum growth temperature experiments. In the sugar fermentation experiment, strains FM1-1, FM1-2, FM1-4 and FM1-8 were unable to ferment glucose, galactose, sucrose, maltose, lactose, raffinose and trehalose. Except glucose, FM1-3, FM3-3 and FM3-7 cannot ferment other sugars. FM1-10, FM1-12, FM2-12 and FM3-2 have different sugar fermentation conditions (Table 2). In addition to various organic acid salts as the sole carbon source, including lactate, succinate and citrate, they also assimilated a wide range of carbon sources (Table 3). They assimilated differently on various nitrogen sources, with FM1-1,FM1-2,FM1-3,FM1-4,FM3-2 being able to utilize sodium nitrate and sodium nitrite, while the rest of the strains were unable to assimilate (Table 4). In addition, in the maximum temperature growth experiment, it was found that all strains except FM1-4 and FM1-8 could grow at $35^{\circ} \mathrm{C}$ (Table 5). According to the morphological, physiological and biochemical characteristics of the above isolate combined with the "Characteristics and Identification Manual of Yeast"(Kurtzman and Cletus 2011; Kurtzman et al. 2011), it can be preliminarily judged that the FM1-1 strain is Aureobasidium, the FM1-2 strain is Naganishia, and the FM1-3 strain is Barnettozyma, Strains FM1-4 and FM1-8 belong to the genus Rhodotorula, strain FM1-10 belongs to the genus Zygosaccharomyces, strain FM1-12 belongs to the genus Saccharomyces, strain FM2-10 belongs to the 
genus Candia, strain FM3-1 belongs to the genus Wickerhamomyces, FM3-3 and The FM3-7 strain belongs to the genus Pichia.

\section{Molecular biology identification and phylogenetic tree construction}

Sequence analysis of 26S rDNA D1/D2 region was performed on yeast isolates in honey. Amplification of the NL1 and NL4 regions produced 400 to 600 bp fragments. According to the sequencing results, submit to the GenBank database of the National Center for Biotechnology Information (NCBI), and use the basic local alignment search tool (BLAST) for homology search, similarity $>99 \%$ was applied to the specieslevel identification of isolates (Fang et al. 2012). Identified strains represented 11 species belonging to 9 genera, namely Aureobasidium, Naganishia, Barnettozyma, Rhodotorula, Zygosaccharomyces, Saccharomyces, Wickerhamomyces, Candia and Pichia. (Table 1). The main species are designated as Zygosaccharomyces ssiamensis, Aureobasidium pullulans and Naganishia albida. Among the 35 identified yeast strains, 24 belonged to Basidiomycetes (approximately 68.6\%), while the remaining 11 strains were designated as Ascomycetes (approximately 31.4\%). The phylogenetic tree constructed based on the 26S rDNA D1/D2 region further confirmed its taxonomic status (Figure 4).

\section{Yeast community structure composition}

Yeast communities from different sources show different species diversity. The majority of strains isolated from oleaster honey are Z.siamensis and N. albida, a colony frequency of $33.33 \%$ and $26.67 \%$; the strains isolated from sunflower honey are mainly Z.siamensis, with a colony frequency of $76.92 \%$. The main strain isolated from honey of various flowers are also Z.siamensis, with a colony frequency of $42.86 \%$. Although Z.siamensis is the dominant strain in the three honey, it is more common in sunflower honey. It is worth mentioning that there are rich types of yeasts in oleaster honey, including 7 species, including A.pullulans, C.albidus, B.californica, R.glutinis, Z.siamensis, R.kratochvilovae, R.dioboratum; There are only 3 species in Shihezi sunflower honey, while there are 5 species in Yili honey of various flowers (Table 6).

\section{Discussion}

Analysis of yeast diversity in wild honey from Xinjiang by the Illumina MiSeq cloud platform with on-line sequencing revealed the presence of abundant yeast resources in honey. Among three honey containing 40 genera and 148 species, the phylum Stenotrophomonas was the dominant fungal phylum, of which Zygosaccharomyces was the dominant genus followed by Aureobasidium, Pichia, which was consistent with the culturable results. The genus Zygosaccharomyces is commonly found in honey (Sujinan et al. 2011; Fan et al. 2014; Liu et al. 2016) and is resistant to hyperosmolarity. Nils Arneborg found that Zygosaccharomyces bailii was resistant to short-term pHi changes caused by acetic acid(Arneborg et al. 2000). Sugumaran has produced commercially important biodegradable polymers, pullulans, from agricultural waste through Aureobasidium pullulans(Sugumaran et al. 2013). Pichia pastoris can be used for the production of Ganoderma immunomodulatory proteins, industrial enzymes and chemicals(Mao et al. 2019; Zhu et al. 2019). Savitree Limtong isolated a new Pichia sp, from soil and found it to be 
thermotolerant and methylotrophic(Savitree et al. 2005). In the heat map, yeasts of the genera Barnettozyma and Eremothecium are relatively less abundant in honey, and both genera have been reported in the literature to have some functional potential as well. Saha found that Barnettozyma populi Y-12728 selectively produces xylitol from mixed sugar and corn stover hemicellulose hydrolysate(Saha and Kennedy 2020). Nouri can improve ethanol production through improved barnettozyma californicac(Nouri et al. 2018). A new essential oil obtained from the fermentation broth of Eremothecium ashbyii $\mathrm{H} 4565$ by Feng He showed some antibacterial activity(He et al. 2018). All these results indicate that yeast germplasm resources are of great significance to humans.

From the yeast community structure, it can be seen that among different honeys, the difference between yeast diversity in Oleaster honey and sunflower honey is not much, which may be related to the geographical environment, because Shihezi is adjacent to Shawan and has similar environmental conditions. However, the diversity and abundance of yeast in multifloral honey was significantly higher than that in Oleaster honey and sunflower honey, which may be due to the fact that monofloral honey and multifloral honey differ in composition and structure, and the microorganisms on the nectar source are more abundant compared to monofloral honey. Mohammed (Mohammed et al. 2013) reported that the chemical composition of honey produced by different species of bees is different, which is of reference for the above study.

Xinjiang honey, as a traditional natural health product, contains a large number of microbial resources (Herrera et al. 2010; Lenaerts 2017). In the experiment. Three honey products were selected as samples of research materials, a total of 15 strains and 7 species of yeast were isolated from date honey, 13 strains and 3 species of yeast from sunflower honey, and 7 strains and 5 species of yeast from peperomia honey. This may be due to geographical conditions, climatic environment and production technology, and traditional culture can be cultivated. The limitations of the method and other factors have caused differences in the microbial composition of wild honey in different regions. Among 35 yeast strains isolated Zygosaccharomyces was found as the dominant yeast genus, followed by Aureobasidium genus, and all 11 yeast species identified were found in high throughput. Physiological characteristics of Zygosaccharomyces yeast including their ability to ferment sugar, osmotic pressure, resistance to preservatives, and heat tolerance of ascospores are very significant (James and Stratford 2003).

In this study, Illumina MiSeq sequencing technology was applied to discover the complex and diverse structure of the wild honey yeast population in Xinjiang, and several yeast genera such as Clavispora, Holtermanniella, and Filobasidium were detected that were not isolated by traditional methods. This further confirmed that Illumina MiSeq sequencing technology not only broadens the depth of microbial diversity in environmental samples, but also provides a comprehensive picture of the flora structure in the samples and presents the true level of microbial diversity (Haifeng et al. 2017; Wu et al. 2020). In the conventional culture, the selectivity of the medium and the restrictive visual observation of the colonies led to errors in the selection of characteristic colonies, and more species of yeast were not isolated. Although the conventional pure culture method has major limitations, it has the advantage that pure cultures can be isolated, such as the genera Barnettozyma, Saccharomyces and Wickerhamomyces, 
which are relatively low in abundance in high-throughput sequencing, were also isolated in the culturable method. Traditional pure culture methods can not only enrich the strain resources, but also provide resources for the application of microorganisms. Therefore, the effective combination of traditional isolation and culture methods with high-throughput sequencing technology can, on the one hand, systematically reveal the diversity of brewer's yeast in wild honey in Xinjiang, and on the other hand, lay the foundation for the enrichment and exploitation of the yeast strain resource base.

\section{Declarations}

\section{Acknowledgements}

This work was supported by Grants-in-Aid for scientific research from the National Natural Science Foundation of China (31860003).

\section{References}

1. A FL, Carolina G, Betiana H, Carlos B, M GL (2017) Microbiological quality of honey from the Pampas Region (Argentina) throughout the extraction process. Revista Argentina de microbiologia 49. https:// doi:10.1016/j.ram.2016.05.010.

2. Arneborg N, Jespersen L, Jakobsen M (2000) Individual cells of Saccharomyces cerevisiae and Zygosaccharomyces bailii exhibit different short-term intracellular $\mathrm{pH}$ responses to acetic acid. Archives of Microbiology Vol.174:125-128 . https://doi: 10.1007/s002030000185

3. Boundy-Mills K (2006) Methods for Investigating Yeast Biodiversity. Springer Berlin Heidelberg

4. Danila C et al. (2018) Phenolic Compounds in Honey and Their Associated Health Benefits: A Review. Molecules 23:2322. https://doi:10.3390/molecules23092322.

5. Deng H, Han Y, Liu Y, Wei J (2013) Erratum to: Identification of a newly isolated erythritol-producing yeast and cloning of its erythrose reductase genes. Journal of Industrial Microbiology \& Biotechnology 40:399. https://doi:10.1007/s10295-012-1162-5.

6. Devi S, Parihar A, Thakur M, Thakur B, Sharma HK (2021) Antibacterial potential of hive bees honey from Himachal Pradesh, India. Archives of Microbiology Vol.203:1-13 doi: 10.1007/s00203-02102489-y. https://doi: 10.1007/s00203-021-02489-y.

7. Di CM, Ma YB, Dong X, Xiang HA, Bu T, Wang CZ (2016) Physicochemical characterization and Volatile components of multifloral, honey of Apis cerana cerana in Deqin. Apiculture of China 67:4651.

8. Echeverrigaray S et al. (2021) Yeast biodiversity in honey produced by stingless bees raised in the highlands of southern Brazil. International Journal of Food Microbiology 347:109200. https://doi:10.1016/j.ijfoodmicro.2021.109200.

9. Fan J, Han Y, Zhou ZJ, Zhao X, Tan XQ (2014) Isolation and identification of osmophilic yeasts from honey. Science and Technology of Food Industry 35:5. https://doi:10.13386/j.issn1002- 
0306.2014.09.027.

10. Fang $Y$ et al. (2012) Isolation,Purification and Identification of Halotolerant Yeast from Soy Sauce Mash and Its Salt Tolerance. Modern Food Science \& Technology 28:922-926. https:// doi:10.13982/j.mfst.1673-9078.2012.08.045.

11. H Tansel Yalçın 1 BF, Yiğit Terzi 2, Ebru Uyar 3, Fatima Shatila 2 (2021) Isolation and molecular identification of industrially important enzyme producer yeasts from tree barks and fruits. Archives of microbiology Vol.203:1079-1088 doi: 10.1007/s00203-020-02104-6. https://doi: 10.1007/s00203020-02104-6.

12. Haifeng et al. (2017) Bacterial diversity in traditional Jiaozi and sourdough revealed by highthroughput sequencing of 16S rRNA amplicons. LWT - Food Science and Technology. https:// doi:10.1016/j.Iwt.2017.04.007.

13. Hajimohammadi B, Dehghani A, Javadzadeh M, Zandi H, Eslami G (2017) Clostridium botulinum spores and fungal contamination in honeys of Iran. Tolooebehdasht 15:1-9.

14. He F, Li K, Zhang X, Yang Y, Xiang F (2018) Components and antibacterial activity of a novel essential oil from the nutrient broth of Eremothecium ashbyii H4565. LWT- Food Science and Technology 101. https:// doi:10.1016/j.Iwt.2018.11.037.

15. Herrera CM, Canto A, Pozo MI, Bazaga P (2010) Inhospitable sweetness: nectar filtering of pollinatorborne inocula leads to impoverished, phylogenetically clustered yeast communities. Proc Biol 277:747-754. https:// doi:10.1098/rspb.2009.1485.

16. Huang X, Wang SS, Liu YW, Li PP, Yang SG (2020) Yeast in honey and its application in food industry. Apiculture of China 71:3.

17. James SA, Stratford M (2003) Spoilage yeasts with emphasis on the genus Zygosaccharomyces. Yeasts in Food. https:// doi: 10.1533/9781845698485.171.

18. Joseph B, Mindy M, Justin H, Adam Q, Matthew B (2018) Two Novel Strains of Torulaspora delbrueckii Isolated from the Honey Bee Microbiome and Their Use in Honey Fermentation. Fermentation 4:22. https:// doi:10.3390/fermentation4020022.

19. Kurtzman, Cletus P (2011) The yeasts. Elsevier:xiii. https:// doi:10.1016/B978-0-444-52149-1.001804.

20. Kurtzman CP, Fell JW, Boekhout T, Robert V (2011) Methods for isolation, phenotypic characterization and maintenance of yeasts. The Yeasts. https://doi:10.1016/B978-0-444-52149-1.00007-0.

21. Lenaerts $M$ (2017) Characterization of microbial communities in floral nectar and assessing their impact on nectar chemistry and performance of biological control agents of pest insects.

22. Li J, Hu WZ, Xu YP (2019) Diversity and Dynamics of Yeasts During Vidal Blanc Icewine Fermentation: A Strategy of the Combination of Culture-Dependent and High-Throughput Sequencing Approaches. other 10. https:// doi:10.3389/fmicb.2019.01588.

23. Liu G et al. (2016) Identification of Zygosaccharomyces mellis strains in stored honey and their stress tolerance. Food science and biotechnology 25:1645-1650.https:// doi:10.1007/s10068-0160253-x. 
24. Liu K, Wei Y, Xiong J, Sun Y, Lei Y (2018) Analysis of diversity of endobiotic yeasts isolated from Locusta migratoria manilensis and Ischiodon scutellaris Fabricius.Guangdong Agric Sci 45:90-95. https:// doi:10.16768/j.issn.1004-874X.2018.09.015

25. Watanabe M, Lee K, Goto K, Kumagai S, Sugita-Konishi Y, and Hara-Kudo Y (2010) Rapid and effective DNA extraction method with bead grinding for a large amount of fungal DNA. Journal of food protection 73.

26. Mao PW, Li LD, Wang YL, Bai XH, Zhou XW (2019) Optimization of the fermentation parameters for the production of Ganoderma lucidum immunomodulatory protein by Pichia pastoris. Preparative Biochemistry \& Biotechnology 50:1-8.https:// doi:10.1080/10826068.2019.1703194.

27. Meo SA, Al-Asiri SA, Mahesar AL, Ansari MJ (2017) Role of honey in modern medicine. Saudi J Biol 24:975-978. https:// doi:10.1016/j.sjbs.2016.12.010.

28. Mohammed et al. (2013) Physicochemical and antioxidant properties of Malaysian honeys produced by Apis cerana, Apis dorsata and Apis mellifera. Bmc Complementary \& Alternative Medicine. https:// doi:10.1186/1472-6882-13-43.

29. Nouri H, Azin M, Mousavi SL (2018) Enhanced ethanol production from sugarcane bagasse hydrolysate with high content of inhibitors by an adapted barnettozyma californica. Environmental Progress \& Sustainable Energy 37. https:// doi:10.1002/ep.12769.

30. P TAC et al. (2003) Starmerella meliponinorum sp. nov., a novel ascomycetous yeast species associated with stingless bees. International journal of systematic and evolutionary microbiology 53. https:// doi: 10.1099/ijs.0.02262-0.

31. Palilu PT, Kasiamdari RS, Ilmi M (2019) Potential Yeast from Indonesian Wild Forest Honey Showing

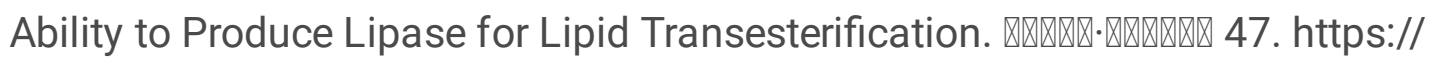
doi:10.4014/mbl.1907.07008.

32. Pallmann CL, Brown JA, Olineka TL, Cocolin L, Mills DA, Bisson LF (2001) Use of WL Medium to Profile Native Flora Fermentations. American Journal of Enology \& Viticulture 52:págs. 198-203.

33. Park YK, Koo MH, Oliveira IMdA (1996) Biochemical Characteristics of Osmophilic Yeasts Isolated from Pollens and Honey. Bioscience, Biotechnology, and Biochemistry Vol.60:1872-1873 doi: 10.1271/bbb.60.1872. https:// doi:10.1271/bbb.60.1872.

34. Rering CC, Rudolph AB, Beck JJ (2021) Pollen and yeast change nectar aroma and nutritional content alone and together, but honey bee foraging reflects only the avoidance of yeast.

Environmental Microbiology. https://doi:10.1111/1462-2920.15528.

35. Roshan N, Rippers T, Locher C, Hammer K (2017) Antibacterial activity and chemical characteristics of several Western Australian honeys compared to manuka honey and pasture honey. Archives of Microbiology Vol.199:347-355.https://doi: 10.1007/s00203-016-1308-3.

36. S GS, Julia A-F, Federico L (2013) Lachancea lanzarotensis sp. nov., an ascomycetous yeast isolated from grapes and wine fermentation in Lanzarote, Canary Islands. International journal of systematic and evolutionary microbiology 63. https:// doi: 10.1099/ijs.0.046029-0. 
37. Saha BC, Kennedy GJ (2020) Optimization of xylitol production from xylose by a novel arabitol limited co-producing Barnettozyma populi NRRL Y-12728. Preparative Biochemistry \& Biotechnology:1-8. https:// doi:10.1080/10826068.2020.1855443.

38. Saksinchai S, Suzuki M, Chantawannakul P, Ohkuma M, Lumyong S (2012) A novel ascosporogenous yeast species, Zygosaccharomyces siamensis, and the sugar tolerant yeasts associated with raw honey collected in Thailand. Fungal Diversity 52. https:// doi:10.1007/s13225011-0115-z.

39. Savitree L, Nantana S, Wichien Y, Hiroya Y, Takashi N, Nobuo K (2005) Pichia thermomethanolica sp. nov., a novel thermotolerant, methylotrophic yeast isolated in Thailand. International journal of systematic and evolutionary microbiology 55. https:// doi 10.1099/ijs.0.63712-0.

40. Shapla UM, Solayman M, Alam N, Khalil MI, Gan SH (2018) 5-Hydroxymethylfurfural (HMF) levels in honey and other food products: effects on bees and human health. Chemistry Central Journal 12:35. https:// doi:10.1186/s13065-018-0408-3.

41. Su Yz, Xie LQ, Wang Q, Abbas A, LI G (2010) SPME-GC-MS Analysis of Volatile Compounds from Four Xin Jiang Monofloral Honey. FOOD SCIENCE 31:293-299

42. Sugumaran KR et al. (2013) Production of pullulan by Aureobasidium pullulans from Asian palm kernel:A novel substrate. Carbohydr Polym 92:697-703.https:// doi:10.1016/j.carbpol.2012.09.062.

43. Sujinan et al. (2011) A novel ascosporogenous yeast species, Zygosaccharomyces siamensis, and the sugar tolerant yeasts associated with raw honey collected in Thailand. Fungal Diversity 52:123139. https:// doi:10.1007/s13225-011-0115-z.

44. úbeda J, Maldonado Gil M, Chiva R, Guillamón JM, Briones A (2014) Biodiversity of nonSaccharomyces yeasts in distilleries of the La Mancha region (Spain). Fems Yeast Research 14. https:// doi:10.1111/1567-1364.12152.

45. Wang C, Wu C, Qiu S (2019) Yeast diversity investigation of Vitis davidii Föex during spontaneous fermentations using culture-dependent and high-throughput sequencing approaches. Food Research International 126. https:// doi:10.1016/j.foodres.2019.108582.

46. Wei ZQ, Zang BL, Xiao FG (2020) Analysis of Volatile Components of Honey from Different Honey Sources. Food Research and Development 41:143-149.

47. Wu X, Jing R, Chen W, Geng X, Liu Y (2020) High-throughput sequencing of the microbial diversity of roasted-sesame-like flavored Daqu with different characteristics. 3 Biotech 10. https:// doi:10.1007/s13205-020-02500-1.

48. Xu JK, Zhang W, Xiao J, Cheng WD, Shi XW (2019) Diversity of yeast in red grape. China Brewing 38:40-45 doi: 10.11882/j.issn.0254-5071.2019.03.009. https:// doi:10.11882/j.issn.02545071.2019.03.009.

49. ZHANG, Kexin, DAl, Shengpei, DONG, Xiaogang (2020) Dynamic Variability in Daily Temperature Extremes and Their Relationships with Large-scale Atmospheric Circulation During 1960-2015 in Xinjiang, China. Chinese Geographical Science v.30:47-62. 
50. Zhang H et al. (2018) Water Bacterial and Fungal Community Compositions Associated with Urban Lakes, Xi'an, China. International Journal of Environmental Research and Public Health 15:469. https:// doi:10.3390/ijerph15030469.

51. Zhang QQ, Li D, Zhang W, Jiang M, Chen XH, Dong MS (2021) Comparative analysis of the bacterial diversity of Chinese fermented sausages using high-throughput sequencing. LWT. https:// doi:10.1111/jam.14648.

52. Zhao WH, Zeng C, Cong YY (2015) Determination of fructose, glucose, sucrose, and maltose in honey by high performance liquid chromatography with refractive index detector. Journal of Food Safety \& Quality 6:4193-4198. https://doi:10.19812/j.cnki.jfsq11-5956/ts.2015.10.071.

53. Zhao Z, Lu X, Chen G, Mao Z, Xie B (2011) [Diversity analysis of archaeal and fungal communities in adjacent cucumber root soil samples in greenhouse by small-subunit rRNA gene cloning]. Chinese Journal of Biotechnology 27:41.

54. Zhu M (2021) Characteristic components of five special monofloral honeys from Northwest China and the gastroprotective effect against ethanol-induced gastric injury. Doctor. https:// doi:10.27405/d.cnki.gxbdu.2021.000002.

55. Zhu SS, Lei YH, Wang C, Wei YM, Sun YF (2021) Patterns of yeast diversity distribution and its drivers in rhizosphere soil of Hami melon orchards in different regions of Xinjiang. BMC Microbiology 21:170. https:// doi:10.1186/s12866-021-02222-1.

56. Zhu T, Sun H, Wang M, Li Y (2019) Pichia pastoris as a Versatile Cell Factory for the Production of Industrial Enzymes and Chemicals: Current Status and Future Perspectives. Biotechnology Journal 14. https:// doi:10.1002/biot.201800694.

\section{Tables}

Table 1 to 6 xlsx are available in the Supplemental Files section.

\section{Figures}




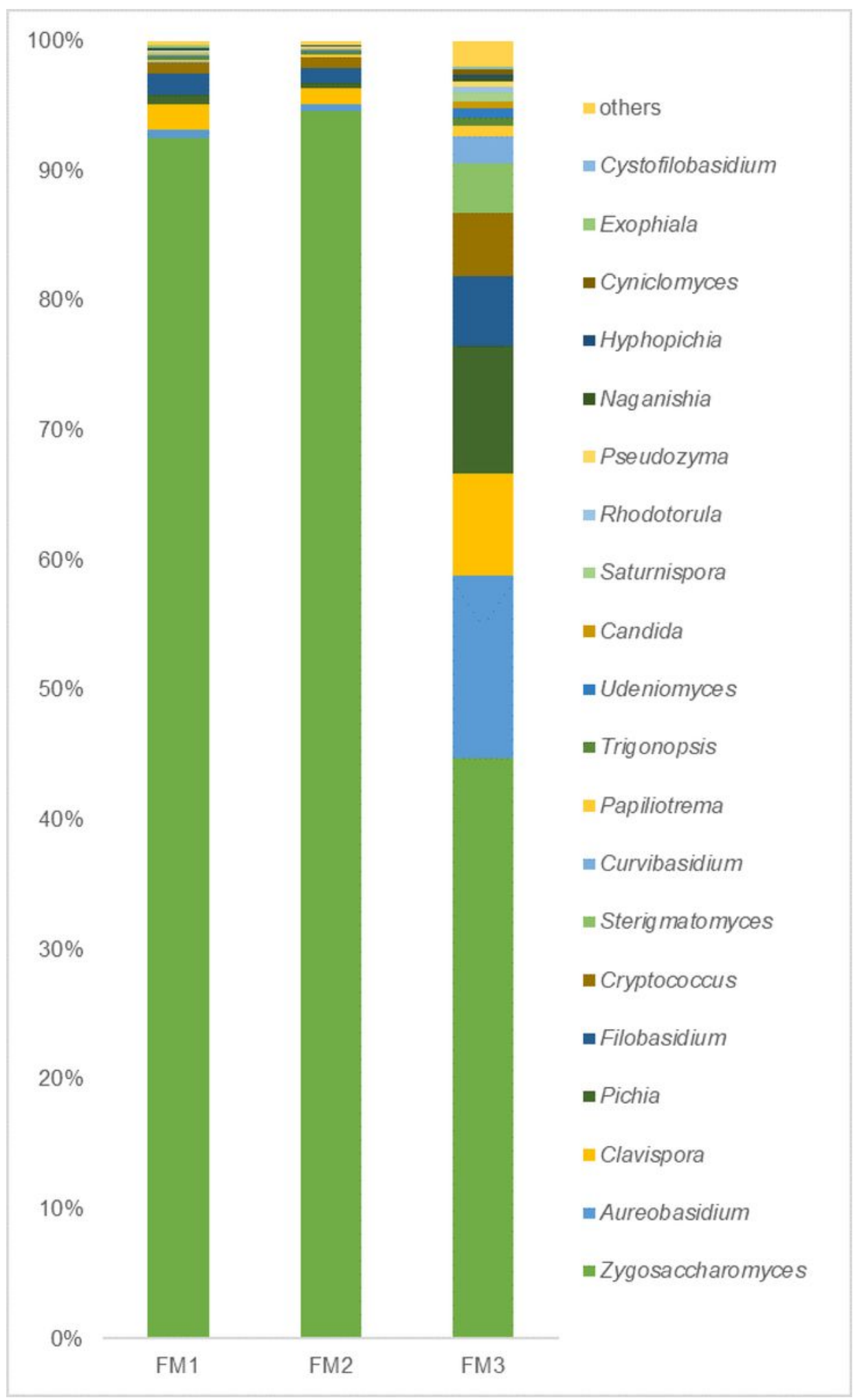

\section{Figure 1}

Relative abundance of species at the level of honey yeast genera.

The top 20 dominant genera were selected from the three honey samples, and the remaining genera were grouped into others, and the relative abundance of species at the yeast genus level was plotted. 


\section{Figure 2}

Heat map of the last 20 OTUs based on relative abundance in the three honey samples. The color intensity in each panel indicates the relative abundance of a genus in each sample, and the abundance was defined as the number of sequences assigned to that taxon divided by the total number of sequences per sample.

\section{Figure 3}

Colonial morphology \left\and cell morphology囚right\of different types of representative strains on WL nutrient agar medium. 


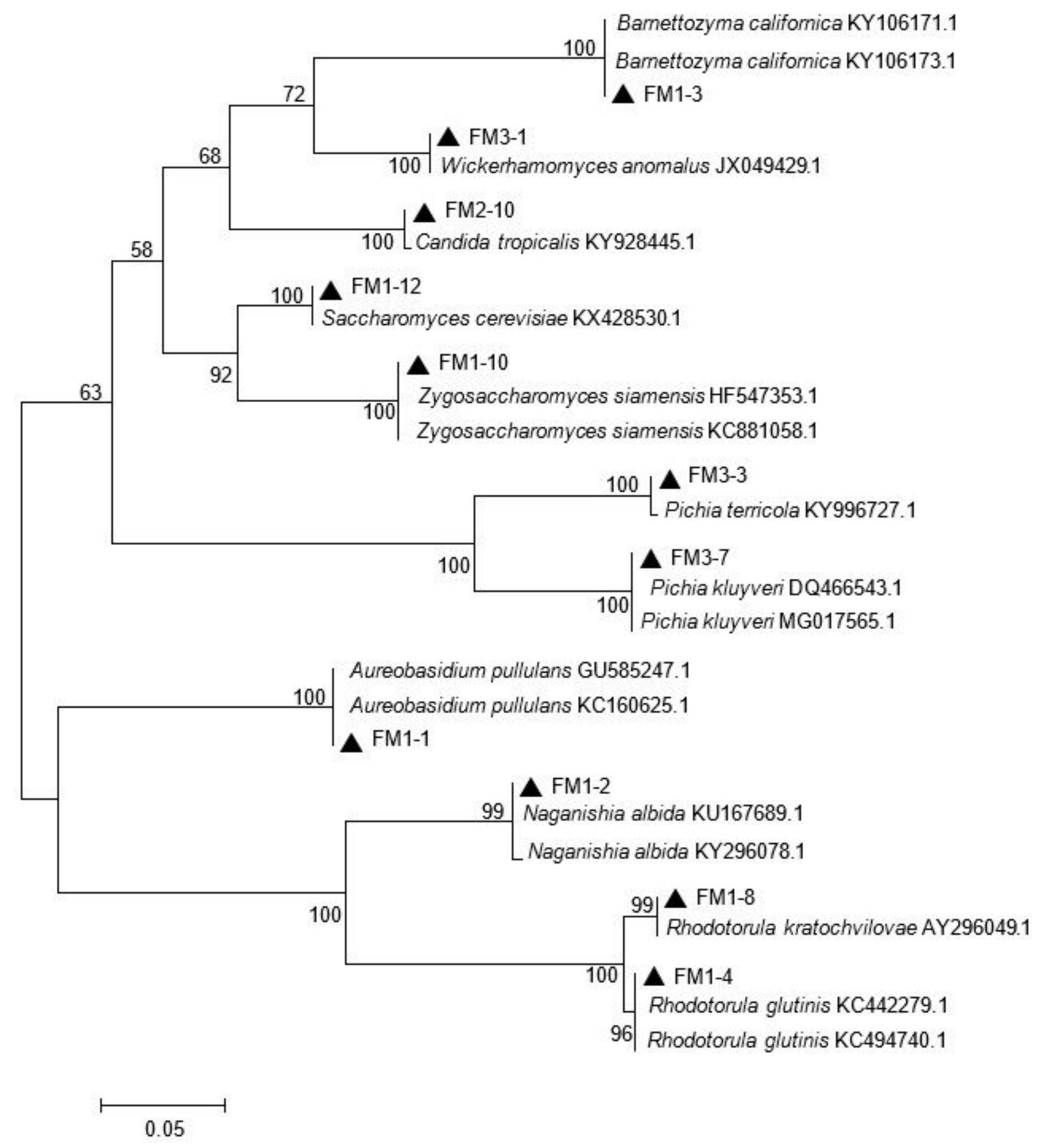

Figure 4

Neighbor-joining phylogenetic tree of the isolates and related reference strains. GenBank accession numbers for the 26S rDNA sequences used in the tree reconstruction are given in the parentheses next to each taxon.

\section{Supplementary Files}

This is a list of supplementary files associated with this preprint. Click to download. 
- Table1.xlsx

- Table2.xlsx

- Table3.xIsx

- Table4.xlsx

- Table5.xlsx

- Table6.xlsx 